\title{
GNormPlus: An Integrative Approach for Tagging Genes, Gene Families, and Protein Domains
}

\author{
Chih-Hsuan Wei, ${ }^{1}$ Hung-Yu Kao, ${ }^{2}$ and Zhiyong $\mathrm{Lu}^{1}$ \\ ${ }^{1}$ National Center for Biotechnology Information (NCBI), 8600 Rockville Pike, Bethesda, MD 20894, USA \\ ${ }^{2}$ Department of Computer Science and Information Engineering, National Cheng Kung University, Tainan 701, Taiwan
}

Correspondence should be addressed to Zhiyong Lu; zhiyong.lu@nih.gov

Received 15 January 2015; Revised 3 April 2015; Accepted 4 April 2015

Academic Editor: Yudong Cai

Copyright (C) 2015 Chih-Hsuan Wei et al. This is an open access article distributed under the Creative Commons Attribution License, which permits unrestricted use, distribution, and reproduction in any medium, provided the original work is properly cited.

\begin{abstract}
The automatic recognition of gene names and their associated database identifiers from biomedical text has been widely studied in recent years, as these tasks play an important role in many downstream text-mining applications. Despite significant previous research, only a small number of tools are publicly available and these tools are typically restricted to detecting only mention level gene names or only document level gene identifiers. In this work, we report GNormPlus: an end-to-end and open source system that handles both gene mention and identifier detection. We created a new corpus of 694 PubMed articles to support our development of GNormPlus, containing manual annotations for not only gene names and their identifiers, but also closely related concepts useful for gene name disambiguation, such as gene families and protein domains. GNormPlus integrates several advanced text-mining techniques, including SimConcept for resolving composite gene names. As a result, GNormPlus compares favorably to other stateof-the-art methods when evaluated on two widely used public benchmarking datasets, achieving $86.7 \%$ F1-score on the BioCreative II Gene Normalization task dataset and 50.1\% F1-score on the BioCreative III Gene Normalization task dataset. The GNormPlus source code and its annotated corpus are freely available, and the results of applying GNormPlus to the entire PubMed are freely accessible through our web-based tool PubTator.
\end{abstract}

\section{Introduction}

With the rapid growth of biomedical literature, textmining or biomedical natural language processing (BioNLP) becomes increasingly important for today's biomedical research [1-6]. BioNLP holds the promise to have computers to read the vast amount of the literature and extract key knowledge about specific topics, such as protein-protein/ drug-drug interactions [7-11], protein functions and transport $[12,13]$, and genetic mutations [14-16]. To accomplish that, the first BioNLP task is often known as named entity recognition (NER): to automatically identify the names of biological entities (e.g., gene/protein) from unstructured texts [17]. Given the central role of gene/proteins in the biomedical research [18], the automatic recognition of gene (note that we use gene and protein interchangeably in this paper) names has received much more attention by the BioNLP researchers [19-26] than other entities such as diseases (e.g., DNorm [27]) and chemicals (e.g., tmChem [28]).

Despite many attempts in the past, the gene NER task remains challenging due to both language variation and ambiguity. First, the same gene is often described in multiple different ways by the authors including the orthographical variation (e.g., "ESR1" and "ESR-1"), morphological variation (e.g., "GHF-1 transcriptional factor" and "GHF-1 transcription factor"), variation with abbreviation (e.g., "estrogen receptor alpha $(\mathrm{ER} \alpha)$ "), and composition mentions (e.g., "BRCA1/2" and "SMADs 1, 5, and 8"). With respect to ambiguity, the first challenge is multispecies (orthologous) gene ambiguity. That is, the same gene name can indicate different concept identifiers depending on its associated organism information (e.g., erbb2 can be either a human gene or mouse gene name). The second ambiguity arises because different genes can share the same name. For example, "AP1 " can refer to either "jun proto-oncogene" (Entrez Gene: 


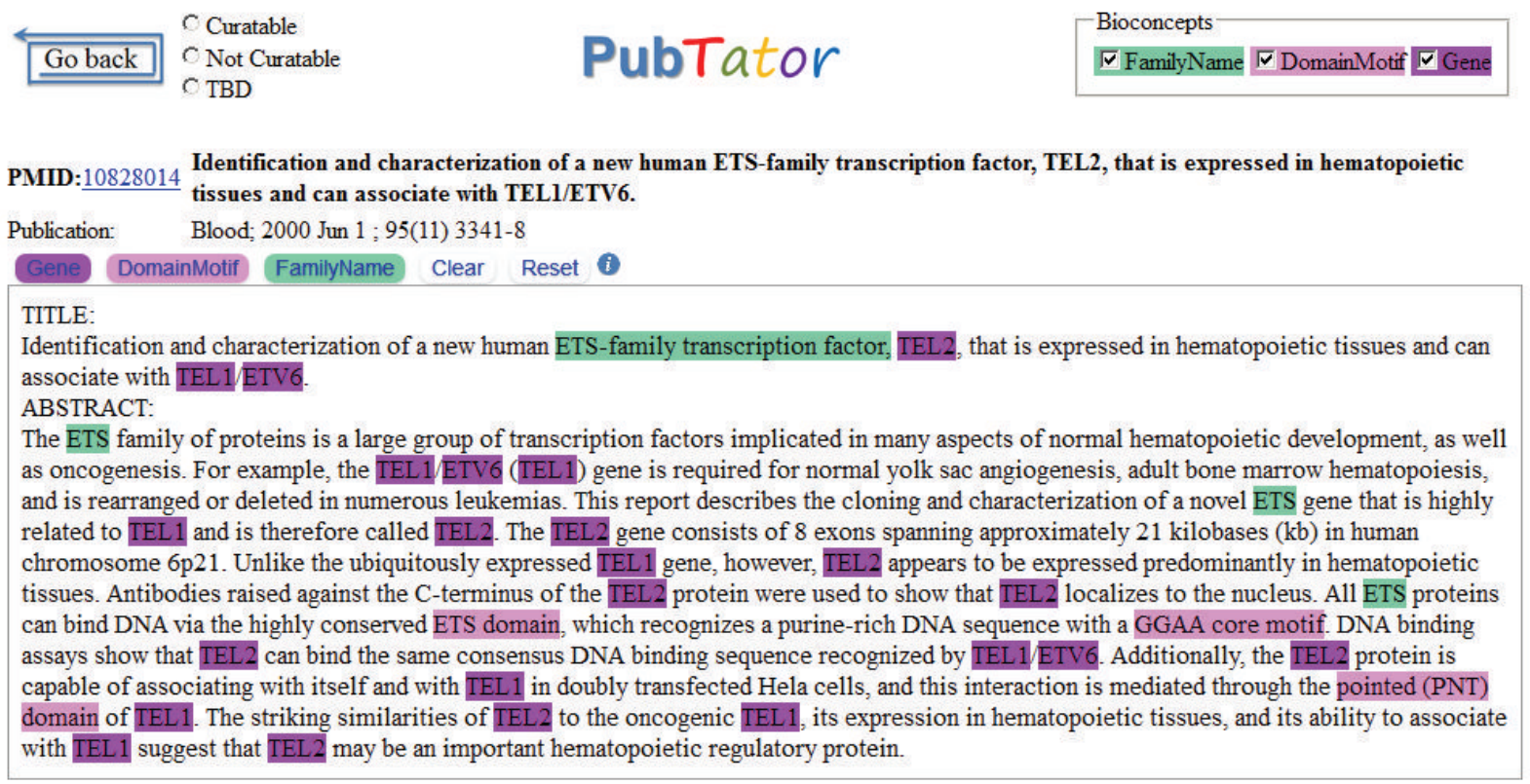

FIGURE 1: A screenshot of gene, gene family, and protein domain annotation of PMID: 10828014 in PubTator.

3725) or "FBJ murine osteosarcoma viral oncogene homolog" (Entrez Gene: 2353).

To advance the state of the art in NER, a number of community-wide shared tasks have been organized [2931] (see Huang and $\mathrm{Lu}, 2015$ [32], for a complete list). In particular, the Critical Assessment of Information Extraction Systems in Biology (BioCreative) has repeatedly organized both gene mention (GM) and gene normalization (GN) tasks where the former task involves finding the occurrence (i.e., string offsets) of gene names in text while the latter typically asks for returning gene concept identifiers per document. In BioCreative I [33] and BioCreative II [7], the GM tasks focused on four species (e.g., human, fly, mouse, and yeast) gene mentions. The best results obtained in the challenges are $83.2 \%$ of $F$-measure in BC I GM task [33] and $88.22 \%$ in BC II GM task [34]. In BioCreative II, the GN task was introduced which asked participants to return human gene/protein concept identifiers given target articles. The best performance in this task was $81.0 \%$ F-measure [7]. In BioCreative III, the GN task was reintroduced with the additional challenges of dealing with full text and multiple species. As a result, the best performance is lower $(46.56 \%$ in F-measure [19]).

As a result of these challenge tasks, a number of annotated corpora were made available to the research community and have, in turn, enabled the development of a number of software tools. For instance, the BioCreative GM corpus was used to build several gene mention taggers, such as AIIAGMT [35], BANNER [36], and BioTagger-GM [37]. However, existing gene corpora (e.g., BioCreative II GM/GN corpora $[29,30])$ are annotated in either mention or document level as they were separately developed. The GM corpus (e.g., [34]) includes mention annotations but not gene identifiers of the target document; the GN corpus contains annotations for the gene identifiers but not their associated mentions. Training a supervised method on some GM data for the GN task is not ideal because different annotation criteria are often used (e.g., GM corpus may include mentions that cannot be mapped to gene identifiers). Thus, we propose developing a corpus that includes both gene mentions and concept identifiers for the same set of articles. To our best knowledge, the newly published IGN corpus [38] is the only other data set that includes both types of annotations. However, we differ from IGN in two main aspects. First, our newly developed corpus consists of more articles (694 versus 543). More importantly, we annotate gene-related concepts separately. That is, we distinguish gene, gene family, and protein domains and treat them as separate classes in our annotation (see Figure 1) as we believe such a distinction can help gene name disambiguation and improve performance. None of the current GM/GN corpora annotates these types separately. For instance, in the BioCreative II GM corpus, gene, protein family, protein domain, DNA, and RNA are all treated as gene mentions.

Past GN systems are unable to distinguish between gene and gene families: they either completely ignored the problem or simply used a protein family name list as filters $[24,25,39$, 40]. However, the filtering strategy does not work once the family mention is not in this list. In this case, the family name becomes false positives in the results. Furthermore, detecting domain names can assist resolving ambiguous gene/protein names. As shown in Figure 2, the TEL1 and TEL2 proteins are both ETS-family transcription factors with the ETS finger domain and GGAA core motif. TEL1 also has the pointed (PNT) domain. When searching for the gene identifier in Entrez Gene, TEL1 can map to two different concepts: ATM serine/threonine kinase (gene ID: 472) and ETS translocation factor variant 6 (gene ID: 2120). But with extracted protein 
TABle 1: The statistic of our gene corpus.

\begin{tabular}{lccc}
\hline Data set & Articles & Gene mentions (gene/family/domains) & Gene identifiers \\
\hline BioCreative II GN training set & 281 & $3,019 / 1,115 / 278$ & 758 \\
BioCreative II GN test set & 262 & $3,233 / 1,252 / 361$ & 928 \\
NLM Citation GIA test collection & 151 & $1,205 / 160 / 17$ & 310 \\
\hline Total & 694 & $7,457 / 2,527 / 656$ & 1996 \\
\hline
\end{tabular}

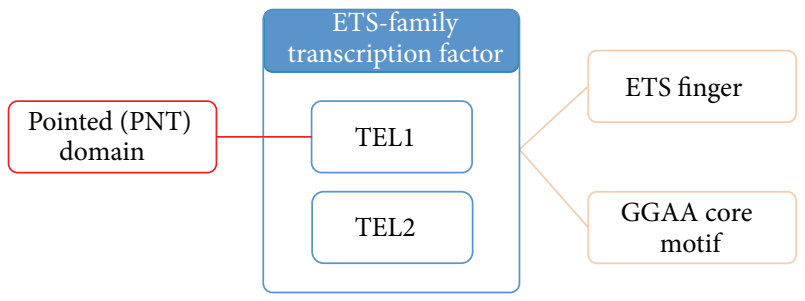

FIGURE 2: Relations between gene, gene family, and protein domains in PMID: 10828014.

domain information, we can infer that in this case ETS translocation factor variant 6 is the correct answer because it is known to be associated with the PNT domain. Besides, the family name "ETS translocation factor" is also helpful to the disambiguation of TEL1/2 because it is included in the gene's official full name.

Taken together, this research makes three major contributions. First, through reannotating two existing corpora, we are the first to build a new corpus that allows the development of new methods for distinguishing different gene-related entities: (gene, gene family, and protein domains). Second, we build a new end-to-end system that includes both GM and GN modules, together with several advanced BioNLP tools (e.g., GenNorm [19], SimConcept [41], SR4GN [42], and Ab3P [43]) for improved performance. Lastly, we show stateof-the-art performance on two separate benchmark data sets.

\section{Materials and Methods}

2.1. Corpus Development. We reannotated two existing gene corpora. The BioCreative II GN corpus is a widely used data set for benchmarking GN tools and includes document level annotations for a total of 543 articles (281 in its training set and 262 in test). The Citation GIA test collection was recently created for gene indexing at the NLM and includes 151 PubMed abstracts with both mention level and document level annotations. They are selected because both have a focus on human genes. For both corpora, we added annotations of gene families and protein domains. For the BioCreative GN corpus, we also added mention level gene annotations. As a result, in our new corpus, there are a total of 694 PubMed articles (see Table 1). PubTator $[44,45]$, a tool developed and evaluated through the BioCreative III Interactive Task [46], was used as our annotation software.

2.2. Method Overview. As shown in Figure 3, our proposed approach includes two main steps: mention recognition and concept normalization, respectively. In the mention recognition step, we developed a new module, together with our previous species recognition system (i.e., SR4GN) to recognize gene and species names and match them accordingly. In concept normalization step, we applied our previous system, GenNorm, combined with a composite mention simplification tool (i.e., SimConcept) and an abbreviation resolution tool (i.e., Ab3P) for optimized performance.

2.3. Mention Recognition Step. In this study, we propose a supervised approach to detect the mentions of gene, gene family, and protein domain from a target input (e.g., PubMed abstracts). We first translate this mention recognition problem as a sequence labeling task. Accordingly, we adapted a probability based sequence detection conditional random fields (CRF) model [47] provided by $\mathrm{CRF}++$ (http://crfpp .googlecode.com/svn/trunk/doc/index.html) library by order 2 model. CRF++ applies L-BFGS [48] which is a QuasiNewton algorithm for large scale numerical optimization problems. We chose BIEO (B: begin, I: inside, E: end, and O: outside) label set for this recognition model. We also used the tokenization module in our previous NER systems (i.e., tmChem [28] and tmVar [15]) here. More specifically, we applied tmVar's tokenization module which splits tokens not only at punctuation (e.g., ".,()+") and spaces, but also at digits and transitions between uppercase and lowercase. For instance, "hTIF1" will be split into three individual tokens "h," "TIF," and "1." We also reused the features in tmChem and tmVar as described below.

(1) General Linguistic Features. We included the original tokens (e.g., genes), stemmed tokens (e.g., gene), and POS tagging result (e.g., "NN"). We also extracted the prefixes and suffixes as features (length: 1 5).

(2) Character Features. Since many gene concepts include letters, digits, and special characters, we therefore detected the number of uppercases, lowercases, letters, digits, and special characters (“;:,.->+_”).

(3) Semantic Features. We defined three types of features to recognize the difference between potential gene mentions and other concepts. We first use the gene vocabulary from ctdbase.org (http://ctdbase.org/ downloads/\#allgenes) to detect those strings which can match gene mentions. In general, literature usually uses abbreviation to describe bioconcepts. We therefore use Ab3P [43] to detect those abbreviation pairs. To help the CRF model to recognize the difference between bioconcepts (e.g., genes, disease, and chemical), we collected a list of semantic tokens for genes (e.g., strains), disease (e.g., "disorder"), 
Recognition step

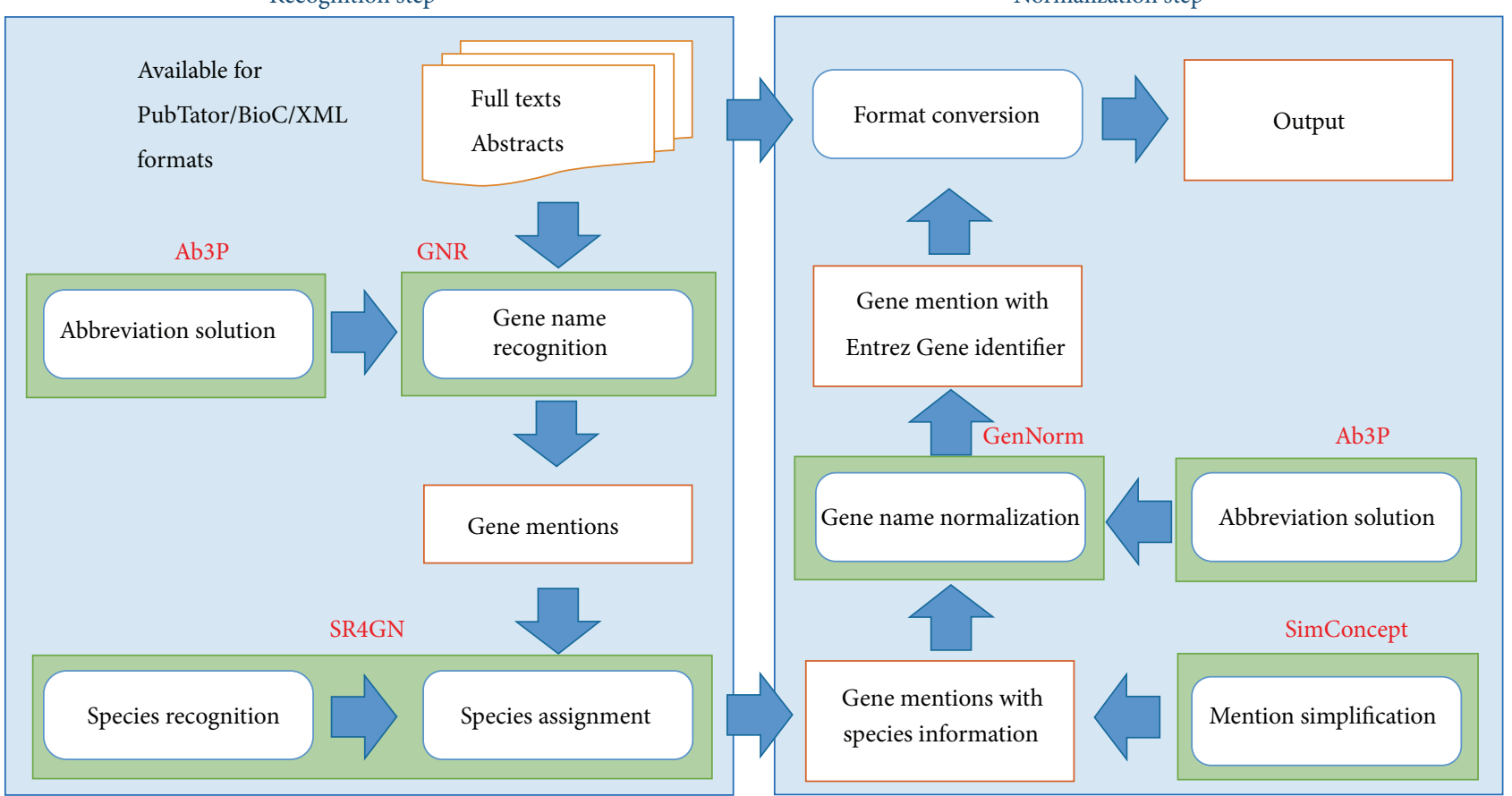

FIGURE 3: The overview of our integration method (GNormPlus). chemical (e.g., "trivial ring"), domain (e.g., "region"), cell (e.g., "cell”), protein symbol (e.g., glutamine), and so forth.

(4) Case Pattern Features. We applied the case pattern features from tmVar [15]. Each token is represented in four simplified forms. Uppercase alphabetic characters are replaced by " $A$ " and lowercase characters are replaced by “a." Likewise, digits (0-9) are replaced by "0." Moreover, we also merged consecutive letters and numbers and generated additional single letter "a" and number " 0 " as features.

(5) Contextual Features. In order to take advantage of contextual information, for a given token we included the dictionary and linguistic features of 3 neighboring tokens from each side.

To best distinguish the three gene-related mention types, gene versus gene family versus protein domains, we applied several postprocessing rules to the CRF results. (1) Set the type by suffix (e.g., "OSBP-related proteins" to family, "LIM1 domain" to domain). (2) If we find a mention (e.g., "TIF1") which is also a prefix of another mention (e.g., "TIFlalpha"), then we set the type of the mention to be gene family. (3) When abbreviation pairs are found, use the mention type of the long form to the sort form (e.g., "TIF1" is tagged as protein family because of its long form "transcriptional intermediary factor 1 family"). (4) If a mention occurs multiple times in an article but is tagged with different types by the CRF module, we then apply the majority rule to determine its final type in the article. For example, if hifl was tagged twice by the $\mathrm{CRF}$ as a gene but as gene family in three times, then all five occurrences of hif1 will be tagged as gene family names.
2.4. Concept Normalization Step. The second step of our system is to map gene mentions to specific concepts in Entrez Gene. To do that, we first applied our previous GN tool, GenNorm [19, 49], which is based on a statistical inference network model via two individual matching strategies (i.e., exact match and bag-of-words match). More specifically, the exact match strategy requires the input mention to be identical to the names in the controlled vocabulary. On the other hand, the bag-of-words approach matches tokens in both input text and target vocabulary. GenNorm achieved the best performance in the BioCreative III GN task [29].

For performance optimization, we also integrated an abbreviation resolution and composite mention simplification tool in this step. First, we applied Ab3P [43] to extract the long form and short form abbreviation pairs. When the short form and long form map to different gene candidates, we typically infer the candidate gene of long form to short form for improved performance. SimConcept [41] was used to identify and resolve composite named entities, where a single span refers to more than one concept (e.g., BRCA1/2). Most past NER studies have either ignored this issue, used simple ad hoc rules, or only handled coordination ellipsis, which is only one of the many types of composite mentions studied in this work. SimConcept was shown to successfully tag individual entities from composite mentions.

\section{Evaluation and Results}

The first evaluation is a species-specific experiment where only human genes are considered. In this evaluation, we trained our system using both BioCreative II GN training set and NLM Citation GIA test collection and tested it on 
TABLE 2: The evaluation of human species gene normalization.

\begin{tabular}{|c|c|c|c|c|}
\hline Methods & Precision & Recall & $F$-measure & System availability \\
\hline Our approach (GNormPlus) & $87.1 \%$ & $86.4 \%$ & $86.7 \%$ & Open source \\
\hline GenNorm [19] + AIIA-GMT [35] & $78.9 \%$ & $81.4 \%$ & $80.1 \%$ & $\begin{array}{l}\text { GenNorm is open source } \\
\text { but AIIA-GMT is no longer } \\
\text { available }\end{array}$ \\
\hline GNAT [23] & $90.7 \%$ & $82.4 \%$ & $86.4 \%$ & Open source \\
\hline GeNO [24] & $87.8 \%$ & $85.0 \%$ & $86.4 \%$ & $\mathrm{~N} / \mathrm{A}$ \\
\hline Hu et al., 2012 [40] & $83.5 \%$ & $82.5 \%$ & $83.0 \%$ & $\mathrm{~N} / \mathrm{A}$ \\
\hline Li et al., 2013 [39] & $88.1 \%$ & $92.3 \%$ & $90.1 \%$ & $\mathrm{~N} / \mathrm{A}$ \\
\hline
\end{tabular}

TABLE 3: The evaluation of cross species gene normalization.

\begin{tabular}{|c|c|c|c|c|c|}
\hline Methods & TAP-5 & TAP-10 & TAP-20 & $F$-measure & System availability \\
\hline Our approach (GNormPlus) & $33.3 \%$ & $36.7 \%$ & $36.7 \%$ & $50.1 \%$ & Open source \\
\hline GenNorm [42] + AIIA-GMT [23] & $32.8 \%$ & $35.5 \%$ & $35.5 \%$ & $46.9 \%$ & $\begin{array}{l}\text { GenNorm is open source } \\
\text { but AIIA-GMT is no longer } \\
\text { available }\end{array}$ \\
\hline GeneTuKit [22] & $29.7 \%$ & $31.4 \%$ & $32.5 \%$ & - & Open source \\
\hline Kuo et al. [21] & $21.4 \%$ & $25.1 \%$ & $25.1 \%$ & $30.6 \%$ & N/A \\
\hline Tsai et al. [20] & $19.0 \%$ & $22.9 \%$ & $23.9 \%$ & - & N/A \\
\hline
\end{tabular}

the BioCreative II GN test set. As shown in Table 2, we compared GNormPlus with several previously reported systems, including our previous system, GenNorm [19]. The default setting of GenNorm uses AIIA-GMT [35] for gene mention recognition. AIIA-GMT is one of the high-performing gene mention recognition tools and provided web API service. Unfortunately, AIIA-GMT is no longer available since 2013.

In the second experiment (see Table 3), we evaluate GNormPlus in multispecies gene normalization using the BioCreative III GN task data set. In this evaluation, we used the whole set of 694 articles for system training. As can be seen, our proposed method significantly outperforms previously published results in both standard $F$-measure and the task-specific TAP-k measures. The new system also outperforms our previous GenNorm tool by a significant margin.

\section{Discussion and Conclusion}

To assess the impact of using multiple gene-related mention types (i.e., gene versus family versus domain), we built a baseline model where all three types were treated as one. As shown in Table 4, the proposed multitype scheme significantly boosted the final GN performance as shown in this comparison.

Despite our best efforts, errors remain in our tagging results. Based on our results on the BioCreative II GN test set, we performed an error analysis including 127 false positive (FP) errors and 87 false negatives. In order to better understand the causes of different errors, we first separated the 214 errors by the GM step and GN step where the former accounts for $53 \%$ and the latter $47 \%$. Among the errors in the GM step, many are due to gene/family/domain mention type
TABLE 4: The comparison of different mention recognition training corpus.

\begin{tabular}{lccc}
\hline $\begin{array}{l}\text { Gene mention type } \\
\text { scheme }\end{array}$ & Precision & Recall & $F$-measure \\
\hline Gene/family/domain & $87.1 \%$ & $86.4 \%$ & $86.7 \%$ \\
Single gene type only & $78.4 \%$ & $79.2 \%$ & $78.8 \%$ \\
\hline
\end{tabular}

confusion (e.g., assigning gene mentions to family/domain or assigning family/domain mentions to genes). Some gene mentions (e.g., TGF-beta) are particularly confusing when they refer to genes in some articles but to family/domain in other articles. In the GN step, failure in disambiguation is a frequent error (17.3\%). A number of gene mentions can be associated with multiple identifiers. With only limited information in the abstract, sometimes it is very difficult to disambiguate and assign genes with correct identifiers. Another $8.9 \%$ of the errors are due to deficiencies of the gene name dictionary. Overall, as can be seen in Table 5, both the GM and GN results are important to the final performance.

To conclude, we developed GNormPlus: an end-to-end gene recognition system which handles both GM and GN tasks. By integrating several advanced BioNLP tools (i.e., GenNorm, SR4GN, Ab3P, and SimConcept), GNormPlus achieved competitive results in our two benchmarking experiments when compared with the state of the art. Unlike our previous GenNorm system that relies on AIIA-GMT, GNormPlus is a stand-alone open source tool with no dependence on external tools (freely available at http://www .ncbi.nlm.nih.gov/CBBresearch/Lu/Demo/tmTools/\#GNormPlus). GNormPlus is made interoperable with other BioCcompatible BioNLP tools. For convenience, we have also 
TABLE 5: The frequency of false negative and positive errors of GNormPlus.

\begin{tabular}{|c|c|c|c|c|}
\hline & FN & $\mathrm{FP}$ & Total & Percentage \\
\hline \multicolumn{5}{|l|}{ Gene mention (GM) recognition } \\
\hline Gene/family/domain mention type confusion & 38 & 18 & 56 & $27.1 \%$ \\
\hline Wrong boundary or missed gene mention & 18 & 18 & 36 & $17.4 \%$ \\
\hline Not a gene mention & 0 & 15 & 15 & $7.3 \%$ \\
\hline \multicolumn{5}{|l|}{ Gene normalization (GN) } \\
\hline Wrong gene identifier due to ambiguity & 19 & 18 & 37 & $17.9 \%$ \\
\hline Insufficiency of the gene name dictionary & 19 & 0 & 19 & $9.2 \%$ \\
\hline Not annotated in the gold standard & 0 & 17 & 17 & $8.2 \%$ \\
\hline Nonhuman genes found & 0 & 11 & 11 & $5.3 \%$ \\
\hline Others & 13 & 3 & 16 & $7.7 \%$ \\
\hline
\end{tabular}

applied GNormPlus to PubMed and stored its results in PubTator (http://www.ncbi.nlm.nih.gov/CBBresearch/Lu/ Demo/PubTator/) so that users can readily access gene data via PubTator. In the future, we plan to explore its applications in real-world uses such as biocuration [50] and also investigate the automatic recognition of other gene-related biological entities such as microRNAs [51].

\section{Conflict of Interests}

The authors declare that there is no conflict of interests regarding the publication of this paper.

\section{Acknowledgments}

The authors would like to thank Robert Leaman for his proofreading of the paper. This research was supported by the NIH Intramural Research Program, National Library of Medicine.

\section{References}

[1] Z. Lu, "PubMed and beyond: a survey of web tools for searching biomedical literature," Database, vol. 2011, Article ID baq036, 2011.

[2] P. Zweigenbaum, D. Demner-fushman, H. Yu, and K. B. Cohen, "Frontiers of biomedical text mining: current progress," Briefings in Bioinformatics, vol. 8, no. 5, pp. 358-375, 2007.

[3] A. Rzhetsky, M. Seringhaus, and M. Gerstein, "Seeking a new biology through text mining," Cell, vol. 134, no. 1, pp. 9-13, 2008.

[4] H. Shatkay and R. Feldman, "Mining the biomedical literature in the genomic era: an overview," Journal of Computational Biology, vol. 10, no. 6, pp. 821-855, 2003.

[5] D. Rebholz-Schuhmann, H. Kirsch, and F. Couto, "Facts from text-is text mining ready to deliver?" PLoS Biology, vol. 3, no. 2, article e65, 2005.

[6] S. Ananiadou, D. B. Kell, and J.-I. Tsujii, "Text mining and its potential applications in systems biology," Trends in Biotechnology, vol. 24, no. 12, pp. 571-579, 2006.

[7] M. Krallinger, F. Leitner, C. Rodriguez-Penagos, and A. Valencia, "Overview of the protein-protein interaction annotation extraction task of BioCreative II," Genome Biology, vol. 9, no. 2, article S4, 2008.
[8] M. Krallinger, M. Vazquez, F. Leitner et al., "The Protein-Protein Interaction tasks of BioCreative III: classification/ranking of articles and linking bio-ontology concepts to full text," BMC Bioinformatics, vol. 12, supplement 8, article S3, 2011.

[9] W. A. Baumgartner Jr., Z. Lu, H. L. Johnson et al., "Concept recognition for extracting protein interaction relations from biomedical text," Genome Biology, vol. 9, supplement 2, article S9, 2008.

[10] I. Segura-Bedmar, P. Martínez, and M. Herrero-Zazo, "Lessons learnt from the DDIExtraction-2013 shared task," Journal of Biomedical Informatics, vol. 51, pp. 152-164, 2014.

[11] I. Segura-Bedmar, P. Martínez, and C. de Pablo-Sánchez, "Using a shallow linguistic kernel for drug-drug interaction extraction," Journal of Biomedical Informatics, vol. 44, no. 5, pp. 789-804, 2011.

[12] J. Gobeill, E. Pasche, D. Vishnyakova, and P. Ruch, "Closing the loop: from paper to protein annotation using supervised Gene Ontology classification," Database, vol. 2014, Article ID bau088, 2014.

[13] Y. Mao, K. Van Auken, D. Li et al., "Overview of the gene ontology task at BioCreative IV," Database, vol. 2014, Article ID bau086, 2014.

[14] A. J. Yepes and K. Verspoor, "Mutation extraction tools can be combined for robust recognition of genetic variants in the literature," F1000Research, vol. 3, article 18, 2014.

[15] C.-H. Wei, B. R. Harris, H.-Y. Kao, and Z. Lu, "TmVar: a text mining approach for extracting sequence variants in biomedical literature," Bioinformatics, vol. 29, no. 11, pp. 1433-1439, 2013.

[16] E. Doughty, A. Kertesz-Farkas, O. Bodenreider et al., "Toward an automatic method for extracting cancer- and other diseaserelated point mutations from the biomedical literature," Bioinformatics, vol. 27, no. 3, Article ID btq667, pp. 408-415, 2011.

[17] W. A. Baumgartner Jr., Z. Lu, H. L. Johnson et al., "An integrated approach to concept recognition in biomedical text," in Proceedings of the 2nd BioCreative Challenge Evaluation Workshop, pp. 257-271, Centro Nacional de Investigaciones Oncologicas (CNIO), Madrid, Spain, 2007.

[18] R. I. Dogan, G. C. Murray, A. Névéol, and Z. Lu, "Understanding PubMed user search behavior through log analysis," Database, vol. 2009, Article ID bap018, 2009.

[19] C.-H. Wei and H.-Y. Kao, "Cross-species gene normalization by species inference," BMC Bioinformatics, vol. 12, supplement 8, article S5, 2011.

[20] R. T. Tsai and P.-T. Lai, "Multi-stage gene normalization for fulltext articles with context-based species filtering for dynamic 
dictionary entry selection," BMC Bioinformatics, vol. 12, supplement 8, article S7, 2011.

[21] C.-J. Kuo, M. H. T. Ling, and C.-N. Hsu, "Soft tagging of overlapping high confidence gene mention variants for crossspecies full-text gene normalization," BMC Bioinformatics, vol. 12, 8, article S6, 2011.

[22] M. Huang, J. Liu, and X. Zhu, "GeneTUKit: a software for document-level gene normalization," Bioinformatics, vol. 27, no. 7, pp. 1032-1033, 2011.

[23] J. Hakenberg, M. Gerner, M. Haeussler et al., "The GNAT library for local and remote gene mention normalization," Bioinformatics, vol. 27, no. 19, Article ID btr455, pp. 2769-2771, 2011.

[24] J. Wermter, K. Tomanek, and U. Hahn, "High-performance gene name normalization with GeNo," Bioinformatics, vol. 25, no. 6 , pp. 815-821, 2009.

[25] J. Hakenberg, C. Plake, R. Leaman, M. Schroeder, and G. Gonzalez, "Inter-species normalization of gene mentions with GNAT," Bioinformatics, vol. 24, no. 16, pp. i126-i132, 2008.

[26] S. van Landeghem, J. Björne, C.-H. Wei et al., "Large-scale event extraction from literature with multi-level gene normalization," PLoS ONE, vol. 8, no. 4, Article ID e55814, 2013.

[27] R. Leaman, R. I. Doğan, and Z. Lu, "DNorm: disease name normalization with pairwise learning to rank," Bioinformatics, vol. 29, no. 22, pp. 2909-2917, 2013.

[28] R. Leaman, C.-H. Wei, and Z. Lu, "tmChem: a high performance approach for chemical named entity recognition and normalization," Journal of Cheminformatics, vol. 7, supplement 1, article S3, 2015.

[29] Z. Lu, H.-Y. Kao, C.-H. Wei et al., “The gene normalization task in BioCreative III," BMC Bioinformatics, vol. 12, 8, article S2, 2011.

[30] A. A. Morgan, Z. Lu, X. Wang et al., "Overview of BioCreative II gene normalization," Genome Biology, vol. 9, supplement 2, article S3, 2008.

[31] L. Hirschman, M. Colosimo, A. Morgan, and A. Yeh, "Overview of BioCreAtIvE task 1B: normalized gene lists," BMC Bioinformatics, vol. 6, supplement 1, article S11, 2005.

[32] C.-C. Huang and Z. Lu, "Community challenges in biomedical text mining over 10 years: success, failure and the future," Briefings in Bioinformatics, 2015.

[33] A. Yeh, A. Morgan, M. Colosimo, and L. Hirschman, "BioCreAtIvE task 1A: gene mention finding evaluation," BMC Bioinformatics, vol. 6, supplement 1, article S2, 2005.

[34] L. Smith, L. K. Tanabe, R. Ando et al., "Overview of BioCreative II gene mention recognition," Genome Biology, vol. 9, no. 2, article S2, 2008.

[35] C.-N. Hsu, Y.-M. Chang, C.-J. Kuo, Y.-S. Lin, H.-S. Huang, and I.-F. Chung, "Integrating high dimensional bi-directional parsing models for gene mention tagging," Bioinformatics, vol. 24, no. 13, pp. i286-i294, 2008.

[36] R. Leaman and G. Gonzalez, "BANNER: an executable survey of advances in biomedical named entity recognition," in Proceedings of the Pacific Symposium on Biocomputing, pp. 652-663, Kohala Coast, Hawaii, USA, January 2008.

[37] M. Torii, Z. Hu, C. H. Wu, and H. Liu, "BioTagger-GM: a gene/protein name recognition system," Journal of the American Medical Informatics Association, vol. 16, no. 2, pp. 247-255, 2009.

[38] H.-J. Dai, J. C.-Y. Wu, and R. T.-H. Tsai, "Collective instancelevel gene normalization on the IGN corpus," PLoS ONE, vol. 8, no. 11, Article ID e79517, 2013.
[39] L. Li, S. Liu, W. Fan, D. Huang, and H. Zhou, "A multistage gene normalization system integrating multiple effective methods," PLoS ONE, vol. 8, no. 12, Article ID e81956, 2013.

[40] Y. Hu, Y. Li, H. Lin, Z. Yang, and L. Cheng, "Integrating various resources for gene name normalization," PLoS ONE, vol. 7, no. 9, Article ID e43558, 2012.

[41] C.-H. Wei, R. leaman, and Z. Lu, "SimConcept: a hybrid approach for simplifying composite named entities in biomedicine," in Proceedings of the ACM Conference on Bioinformatics Computational Biology and Health Informatics, pp. 138-146, ACM, Newport Beach, Calif, USA, 2014.

[42] C.-H. Wei, H.-Y. Kao, and Z. Lu, "SR4GN: a species recognition software tool for gene normalization," PLoS ONE, vol. 7, no. 6, Article ID e38460, 2012.

[43] S. Sohn, D. C. Comeau, W. Kim, and J. W. Wilbur, "Abbreviation definition identification based on automatic precision estimates," BMC Bioinformatics, vol. 9, no. 1, article 402, 2008.

[44] C.-H. Wei, H.-Y. Kao, and Z. Lu, "PubTator: a web-based text mining tool for assisting biocuration," Nucleic Acids Research, vol. 41, pp. W518-W522, 2013.

[45] C.-H. Wei, B. R. Harris, D. Li et al., "Accelerating literature curation with text-mining tools: a case study of using PubTator to curate genes in PubMed abstracts," Database, vol. 2012, Article ID bas041, 2012.

[46] C. N. Arighi, B. Carterette, K. B. Cohen et al., "An overview of the BioCreative 2012 Workshop Track III: interactive text mining task," Database, vol. 2013, Article ID bas056, 2013.

[47] J. Lafferty, A. McCallum, and F. Pereira, "Conditional random fields: probabilistic models for segmenting and labeling sequence data," in Proceedings of the 18th International Conference on Machine Learning (ICML '01), pp. 282-289, ACM, Williamstown, Mass, USA, June-July 2001.

[48] D. C. Liu and J. Nocedal, "On the limited memory BFGS method for large scale optimization," Mathematical Programming B, vol. 45, no. 3, pp. 503-528, 1989.

[49] C.-H. Wei, I.-C. Huang, Y.-Y. Hsu, and H.-Y. Kao, "Normalizing biomedical name entities by similarity-based inference network and de-ambiguity mining," in Proceedings of the 9th IEEE International Conference on Bioinformatics and Bioengineering, pp. 461-466, Taichung, Taiwan, June 2009.

[50] Z. Lu and L. Hirschman, "Biocuration workflows and text mining: overview of the BioCreative 2012 Workshop Track II," Database, vol. 2012, Article ID bas043, 2012.

[51] B. Xie, Q. Ding, H. Han, and D. Wu, "miRCancer: a microRNAcancer association database constructed by text mining on literature," Bioinformatics, vol. 29, no. 5, pp. 638-644, 2013. 

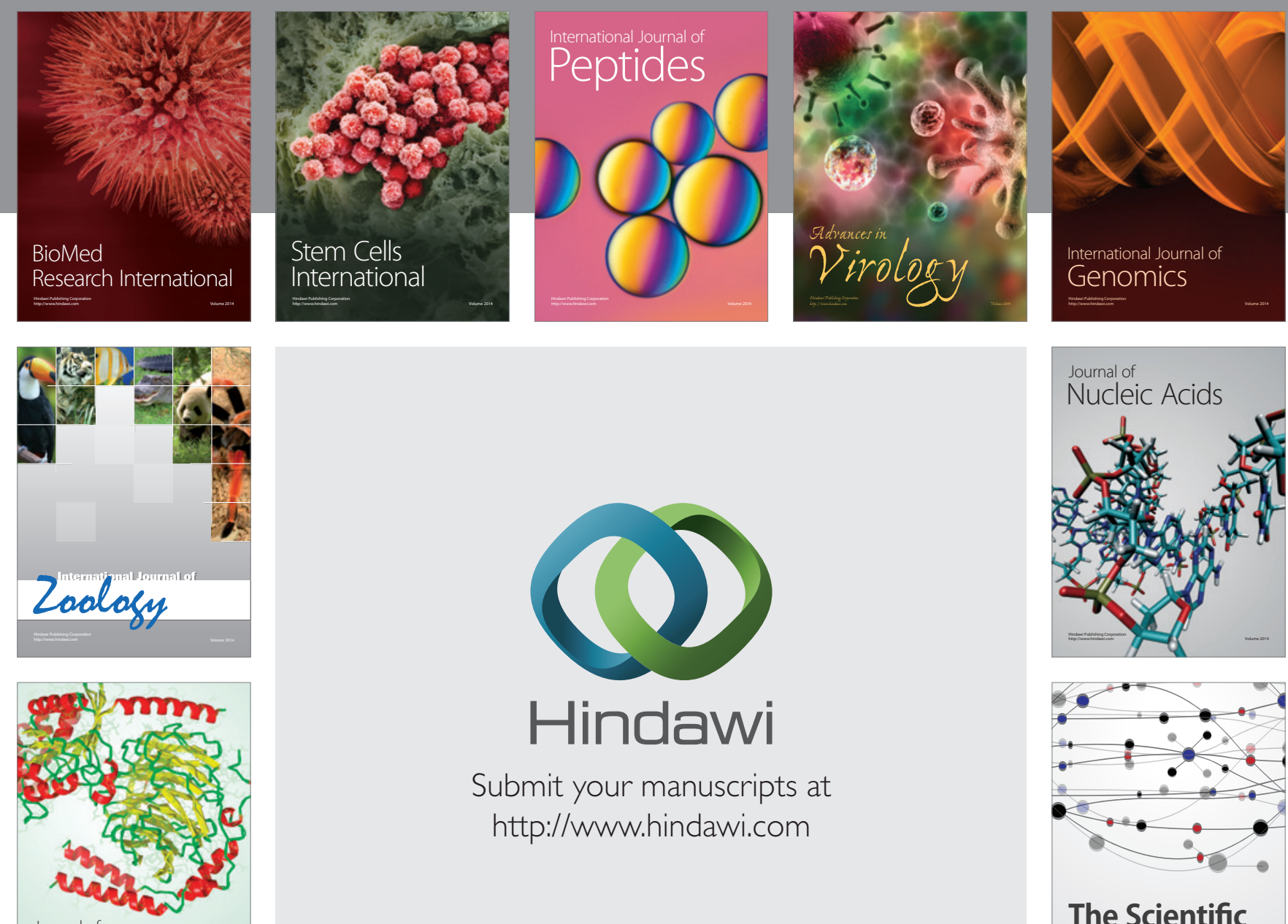

Submit your manuscripts at

http://www.hindawi.com

Journal of
Signal Transduction
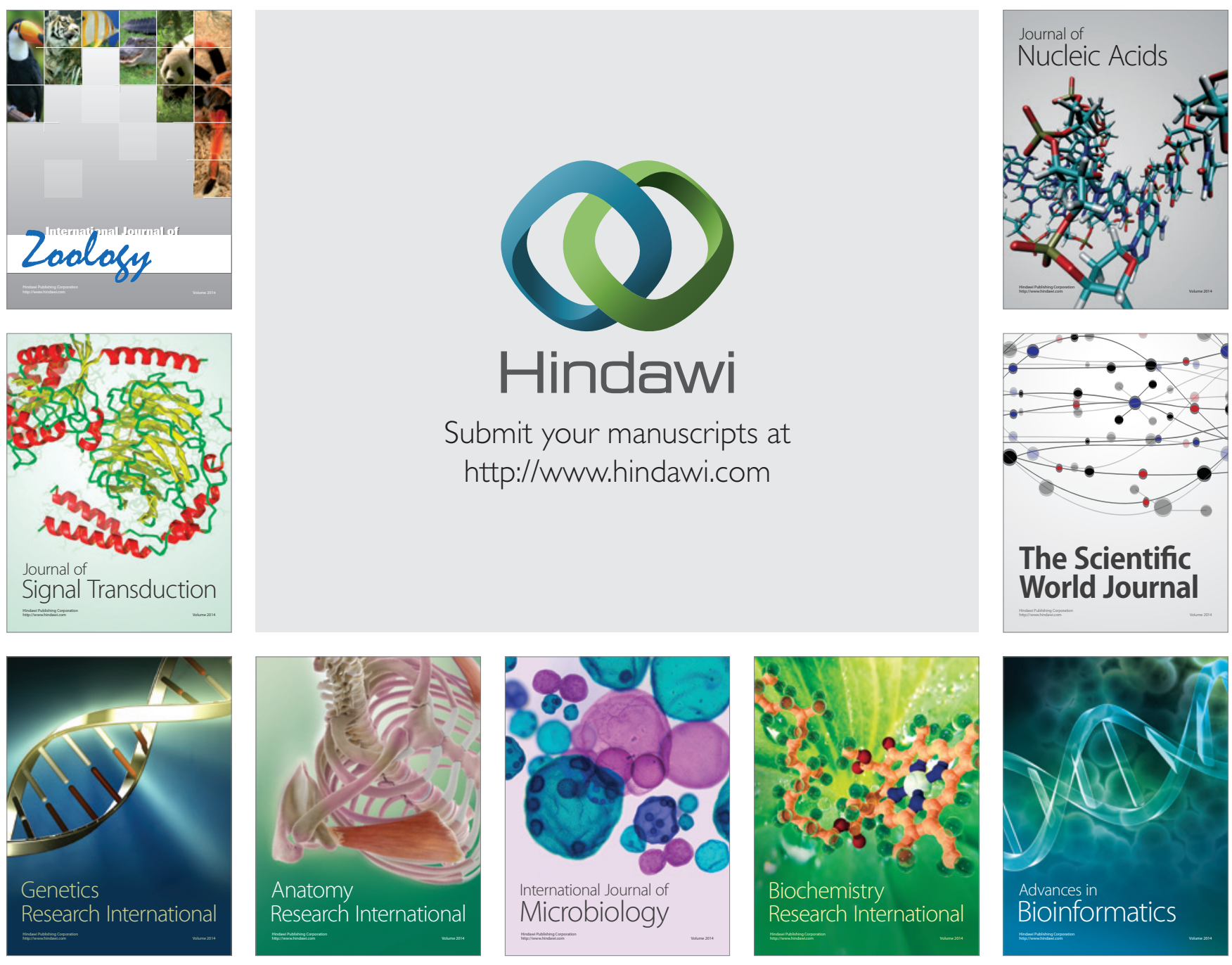

The Scientific World Journal
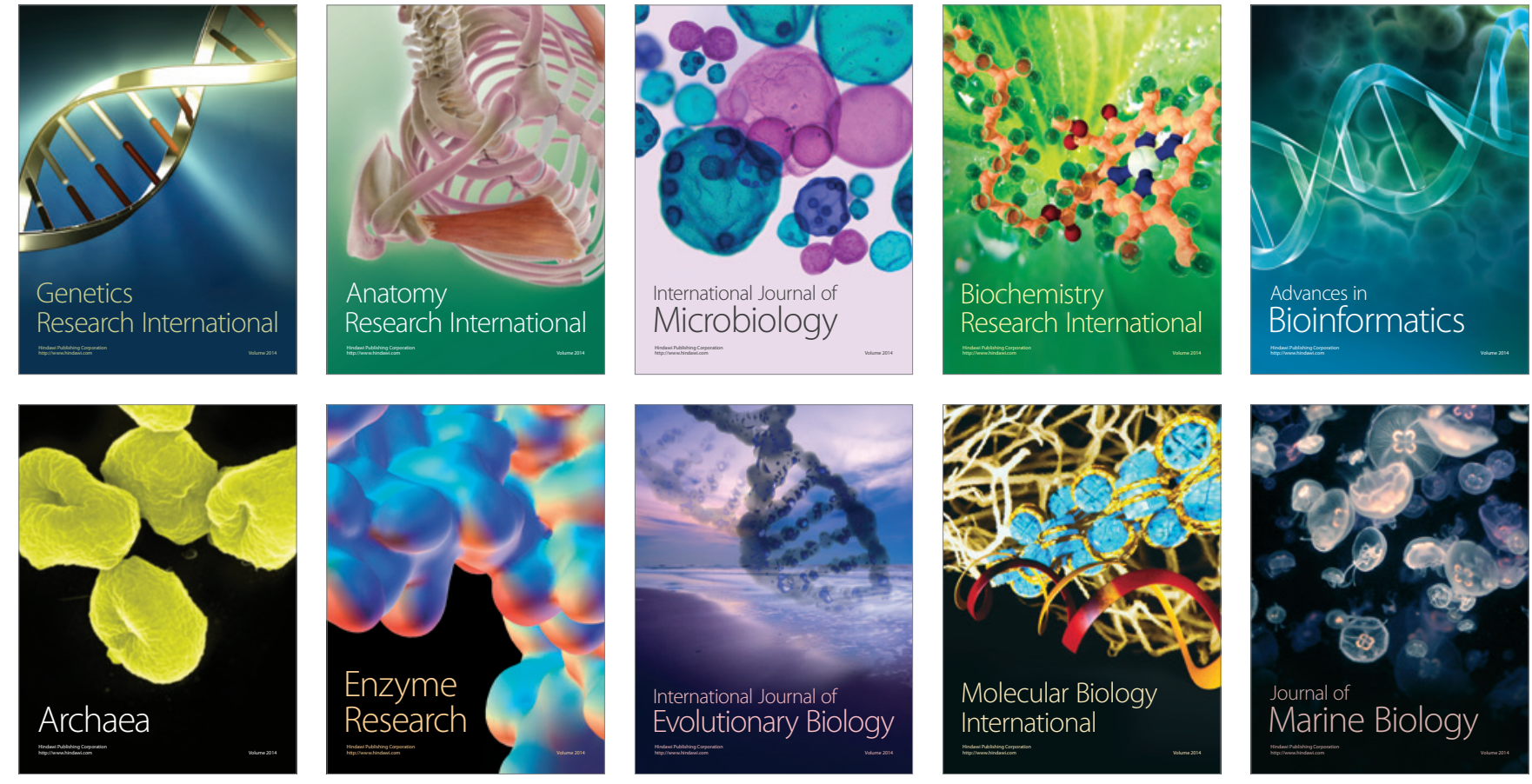